Illinois State University

ISU ReD: Research and eData

Faculty Publications - Biological Sciences

Biological Sciences

2021

\title{
Dataset for Response of the Invasive Alliaria Petiolata to Extreme Temperatures and Drought
}

\author{
Roger C. Anderson \\ Illinois State University \\ Rebecca Anderson \\ Illinois State University \\ Jonathan T. Bauer \\ Miami University - Oxford \\ Christopher Loebach \\ Kapur \& Associates, Inc. \\ Alicia Mullarkey \\ Driftless Area Wetlands Centre
}

See next page for additional authors

Follow this and additional works at: https://ir.library.illinoisstate.edu/fpbiosci

Part of the Ecology and Evolutionary Biology Commons

\section{Recommended Citation}

Anderson, Roger C.; Anderson, Rebecca; Bauer, Jonathan T.; Loebach, Christopher; Mullarkey, Alicia; and Engelhardt, Megan, "Dataset for Response of the Invasive Alliaria Petiolata to Extreme Temperatures and Drought" (2021). Faculty Publications - Biological Sciences. 31.

https://ir.library.illinoisstate.edu/fpbiosci/31

This Data is brought to you for free and open access by the Biological Sciences at ISU ReD: Research and eData. It has been accepted for inclusion in Faculty Publications - Biological Sciences by an authorized administrator of ISU ReD: Research and eData. For more information, please contact ISUReD@ilstu.edu. 


\section{Authors}

Roger C. Anderson, Rebecca Anderson, Jonathan T. Bauer, Christopher Loebach, Alicia Mullarkey, and Megan Engelhardt 


\section{SUPPORTING INFORMATION}

Response of the invasive Alliaria petiolata to extreme temperatures and drought

Roger C. Anderson, M. Rebecca Anderson, Jonathan T. Bauer, Christopher Loebach, Alicia Mullarkey, and Megan Engelhardt

\section{Ecosphere}

Appendix S1: Fig. S1

Data is used to generate Fig. S1, which includes densities of rosettes and mature plants from

$2004-2014$

/*Data are rosettes, plotid (plot identification), counts, and quadrats. There are two woods each with two blocks. Quadno are quadrat numbers, numbers 1-11 indicate sample years from 2004-2014. Codes are SAS for PROC GLIMMIX.*/

Data rostrent;

Input plotid woods block quadno @; do $\mathrm{i}=1$ to $11 ; / * 2004-2014 * /$

input time count @ @

count $1=(\operatorname{count}+1)$;

output;

end;

datalines;

$\begin{array}{cccccccccccccccccccccccccc}\mathbf{1} & \mathbf{1} & \mathbf{1} & \mathbf{1} & \mathbf{1} & \mathbf{1} & \mathbf{2} & \mathbf{6} & \mathbf{3} & \mathbf{0} & \mathbf{4} & \mathbf{8} & \mathbf{5} & \mathbf{1} & \mathbf{6} & \mathbf{0} & \mathbf{7} & \mathbf{0} & \mathbf{8} & \mathbf{0} & \mathbf{9} & \mathbf{0} & \mathbf{1 0} & \mathbf{7} & \mathbf{1 1} & \mathbf{0} \\ \mathbf{2} & \mathbf{1} & \mathbf{1} & \mathbf{7} & \mathbf{1} & \mathbf{2} & \mathbf{2} & \mathbf{0} & \mathbf{3} & \mathbf{0} & \mathbf{4} & \mathbf{7} & \mathbf{5} & \mathbf{2} & \mathbf{6} & \mathbf{0} & \mathbf{7} & \mathbf{1} & \mathbf{8} & \mathbf{0} & \mathbf{9} & \mathbf{0} & \mathbf{1 0} & \mathbf{6} & \mathbf{1 1} & \mathbf{1} \\ \mathbf{3} & \mathbf{1} & \mathbf{1} & \mathbf{9} & \mathbf{1} & \mathbf{1} & \mathbf{2} & \mathbf{1 0} & \mathbf{3} & \mathbf{0} & \mathbf{4} & \mathbf{1 0} & \mathbf{5} & \mathbf{4} & \mathbf{6} & \mathbf{0} & \mathbf{7} & \mathbf{0} & \mathbf{8} & \mathbf{0} & \mathbf{9} & \mathbf{0} & \mathbf{1 0} & \mathbf{1} & \mathbf{1 1} & \mathbf{0} \\ \mathbf{4} & \mathbf{1} & \mathbf{1} & \mathbf{1 0} & \mathbf{1} & \mathbf{0} & \mathbf{2} & \mathbf{4} & \mathbf{3} & \mathbf{0} & \mathbf{4} & \mathbf{4} & \mathbf{5} & \mathbf{0} & \mathbf{6} & \mathbf{0} & \mathbf{7} & \mathbf{0} & \mathbf{8} & \mathbf{0} & \mathbf{9} & \mathbf{0} & \mathbf{1 0} & \mathbf{0} & \mathbf{1 1} & \mathbf{1}\end{array}$




\begin{tabular}{|c|c|c|c|c|c|c|c|c|c|c|c|c|c|c|c|c|c|c|c|c|c|c|c|c|c|}
\hline 5 & 1 & 1 & 15 & 1 & 1 & 2 & 4 & 3 & 0 & 4 & 4 & 5 & 4 & 6 & 0 & 7 & 0 & 8 & 1 & 9 & 0 & 10 & 10 & 11 & $\mathbf{0}$ \\
\hline 6 & 1 & 1 & 17 & 1 & 0 & 2 & 14 & 3 & $\mathbf{0}$ & 4 & 11 & 5 & 2 & 6 & 0 & 7 & 0 & 8 & 0 & 9 & 0 & 10 & 5 & 11 & 0 \\
\hline 7 & 1 & 1 & 21 & 1 & 5 & 2 & 7 & 3 & 0 & 4 & 22 & 5 & 2 & 6 & 0 & 7 & 0 & 8 & 1 & 9 & & 10 & 4 & 11 & 3 \\
\hline 8 & 1 & 1 & 23 & 1 & 0 & 2 & 4 & 3 & 0 & 4 & 7 & 5 & 5 & 6 & 0 & 7 & 0 & 8 & 0 & 9 & & 10 & 4 & 11 & 0 \\
\hline 9 & 1 & 1 & 26 & 1 & 6 & 2 & 13 & 3 & 0 & 4 & 10 & 5 & 4 & 6 & 0 & 7 & 1 & 8 & 0 & 9 & & 10 & 15 & 11 & 0 \\
\hline 10 & 1 & 1 & 30 & 1 & 3 & 2 & 6 & 3 & 0 & 4 & 9 & 5 & 6 & 6 & 0 & 7 & 0 & 8 & 1 & 9 & & 10 & 16 & 11 & 2 \\
\hline 11 & 1 & 1 & 31 & 1 & 0 & 2 & 11 & 3 & 0 & 4 & 10 & 5 & 4 & 6 & 0 & 7 & 0 & 8 & 0 & 9 & 0 & 10 & 4 & 11 & 0 \\
\hline 12 & 1 & 1 & 36 & 1 & 2 & 2 & 11 & 3 & 0 & 4 & 4 & 5 & 0 & 6 & 1 & 7 & 0 & 8 & 4 & 9 & & 10 & 20 & 11 & 0 \\
\hline 13 & 1 & 1 & 42 & 1 & 0 & 2 & 0 & 3 & 0 & 4 & 5 & 5 & 0 & 6 & 0 & 7 & 0 & 8 & 0 & 9 & 0 & 10 & 1 & 11 & 0 \\
\hline 14 & 1 & 1 & 44 & 1 & 1 & 2 & 17 & 3 & 0 & 4 & 3 & 5 & 6 & 6 & 0 & 7 & 4 & 8 & 0 & 9 & J & 10 & 44 & 11 & 1 \\
\hline 15 & 1 & 1 & 47 & 1 & 0 & 2 & 6 & 3 & 0 & 4 & 2 & 5 & 7 & 6 & 0 & 7 & 0 & 8 & 1 & 9 & 0 & 10 & 10 & 11 & 0 \\
\hline 16 & 1 & 1 & 48 & 1 & 1 & 2 & 6 & 3 & 0 & 4 & 7 & 5 & 4 & 6 & 0 & 7 & 0 & 8 & 0 & 9 & D & 10 & 5 & 11 & 4 \\
\hline 17 & 1 & 1 & 53 & 1 & 0 & 2 & 7 & 3 & 0 & 4 & 7 & 5 & 7 & 6 & 2 & 7 & 0 & 8 & 6 & 9 & 0 & 10 & 17 & 11 & 4 \\
\hline 18 & 1 & 1 & 56 & 1 & 2 & 2 & 11 & 3 & 0 & 4 & 5 & 5 & 2 & 6 & 0 & 7 & 0 & 8 & 0 & 9 & 0 & 10 & 15 & 11 & 0 \\
\hline 19 & 1 & 1 & 57 & 1 & 0 & 2 & 6 & 3 & $\mathbf{0}$ & 4 & 3 & 5 & 9 & 6 & 0 & 7 & 0 & 8 & 0 & 9 & 0 & 10 & 4 & 11 & 0 \\
\hline 20 & 1 & 1 & 60 & 1 & 1 & 2 & 11 & 3 & 0 & 4 & 11 & 5 & 0 & 6 & 1 & 7 & 0 & 8 & 0 & 9 & 0 & 10 & 7 & 11 & 2 \\
\hline 21 & 1 & 2 & 1 & 1 & 14 & 2 & 6 & 3 & 6 & 4 & 5 & 5 & 19 & 6 & 0 & 7 & 0 & 8 & 3 & 9 & 0 & 10 & 12 & 11 & 6 \\
\hline 22 & 1 & 2 & 7 & 1 & 1 & 2 & 11 & 3 & $\mathbf{0}$ & 4 & 5 & 5 & 8 & 6 & 2 & 7 & $\mathbf{0}$ & 8 & 1 & 9 & 0 & 10 & 5 & 11 & 0 \\
\hline 23 & 1 & 2 & 9 & 1 & 27 & 2 & 8 & 3 & 0 & 4 & 2 & 5 & 5 & 6 & 0 & 7 & 2 & 8 & 5 & 9 & 1 & 10 & 0 & 11 & 10 \\
\hline 24 & 1 & 2 & 10 & 1 & 16 & 2 & 11 & 3 & 0 & 4 & 16 & 5 & 9 & 6 & 6 & 7 & 0 & 8 & 3 & 9 & 0 & 10 & 0 & 11 & 2 \\
\hline 25 & 1 & 2 & 15 & 1 & 0 & 2 & 13 & 3 & 2 & 4 & 3 & 5 & 20 & 6 & 1 & 7 & 0 & 8 & 1 & 9 & 0 & 10 & 1 & 11 & 11 \\
\hline 26 & 1 & 2 & 17 & 1 & 3 & 2 & 6 & 3 & 0 & 4 & 3 & 5 & $\mathbf{3}$ & 6 & 2 & 7 & 0 & 8 & 1 & 9 & 0 & 10 & 0 & 11 & 2 \\
\hline 27 & 1 & 2 & 21 & 1 & 1 & 2 & $\mathbf{0}$ & 3 & 0 & 4 & 5 & 5 & 6 & 6 & 0 & 7 & 2 & 8 & 0 & 9 & 0 & 10 & 2 & 11 & 0 \\
\hline 28 & 1 & 2 & 23 & 1 & 7 & 2 & 7 & 3 & 0 & 4 & 0 & 5 & 6 & 6 & 0 & 7 & 0 & 8 & 1 & 9 & 0 & 10 & 0 & 11 & 2 \\
\hline 29 & 1 & 2 & 26 & 1 & 0 & 2 & 4 & 3 & 1 & 4 & 8 & 5 & 19 & 6 & 0 & 7 & 1 & 8 & 3 & 9 & 2 & 10 & 17 & 11 & 2 \\
\hline 30 & 1 & 2 & 30 & 1 & 0 & 2 & 1 & 3 & 0 & 4 & 9 & 5 & 10 & 6 & 13 & 7 & 1 & 8 & 1 & 9 & (0) & 10 & 17 & 11 & 9 \\
\hline 31 & 1 & 2 & 31 & 1 & 0 & 2 & $\mathbf{0}$ & 3 & 0 & 4 & 16 & 5 & 9 & 6 & 0 & 7 & 1 & 8 & 8 & 9 & 0 & 10 & 5 & 11 & 0 \\
\hline 32 & 1 & 2 & 36 & 1 & 5 & 2 & 2 & 3 & 0 & 4 & 2 & 5 & 3 & 6 & 0 & 7 & 0 & 8 & 13 & 9 & 2 & 10 & 9 & 11 & 1 \\
\hline 33 & 1 & 2 & 42 & 1 & 3 & 2 & 17 & 3 & 0 & 4 & 3 & 5 & 0 & 6 & 1 & 7 & 0 & 8 & $\mathbf{1}$ & 9 & (0 & 10 & 13 & 11 & 6 \\
\hline 34 & 1 & 2 & 44 & 1 & 0 & 2 & 4 & 3 & 0 & 4 & 1 & 5 & 4 & 6 & 4 & 7 & 0 & 8 & $\mathbf{0}$ & 9 & 0 & 10 & 6 & 11 & \\
\hline
\end{tabular}


$\begin{array}{llllllllllllllllllllllllll}35 & 1 & 2 & 47 & 1 & 0 & 2 & 36 & 3 & 1 & 4 & 8 & 5 & 0 & 6 & 3 & 7 & 0 & 8 & 1 & 9 & 0 & 10 & 0 & 11 & 2\end{array}$ $\begin{array}{llllllllllllllllllllllllll}36 & 1 & 2 & 48 & 1 & 3 & 2 & 2 & 3 & 1 & 4 & 2 & 5 & 3 & 6 & 2 & 7 & 0 & 8 & 1 & 9 & 0 & 10 & 1 & 11 & 11\end{array}$ $\begin{array}{llllllllllllllllllllllllll}37 & 1 & 2 & 53 & 1 & 0 & 2 & 4 & 3 & 0 & 4 & 17 & 5 & 8 & 6 & 16 & 7 & 0 & 8 & 27 & 9 & 0 & 10 & 11 & 11 & 1\end{array}$ $\begin{array}{llllllllllllllllllllllllll}38 & 1 & 2 & 56 & 1 & 3 & 2 & 7 & 3 & 0 & 4 & 11 & 5 & 3 & 6 & 2 & 7 & 1 & 8 & 0 & 9 & 0 & 10 & 0 & 11 & 1\end{array}$ $\begin{array}{llllllllllllllllllllllllllll}39 & 1 & 2 & 57 & 1 & 4 & 2 & 27 & 3 & 0 & 4 & 1 & 5 & 6 & 6 & 1 & 7 & 1 & 8 & 3 & 9 & 0 & 10 & 20 & 11 & 7\end{array}$ $\begin{array}{lllllllllllllllllllllllllll}40 & 1 & 2 & 60 & 1 & 0 & 2 & 1 & 3 & . & 4 & 13 & 5 & 20 & 6 & 4 & 7 & 0 & 8 & 0 & 9 & 0 & 10 & 0 & 11 & 0\end{array}$ $\begin{array}{llllllllllllllllllllllllll}41 & 2 & 3 & 1 & 1 & 1 & 2 & 1 & 3 & 0 & 4 & 2 & 5 & 0 & 6 & 22 & 7 & 1 & 8 & 0 & 9 & 0 & 10 & 0 & 11 & 2\end{array}$ $\begin{array}{llllllllllllllllllllllllll}42 & 2 & 3 & 7 & 1 & 0 & 2 & 23 & 3 & 1 & 4 & 4 & 5 & 4 & 6 & 0 & 7 & 0 & 8 & 0 & 9 & 0 & 10 & 0 & 11 & 0\end{array}$ $\begin{array}{llllllllllllllllllllllllllllll}43 & 2 & 3 & 9 & 1 & 7 & 2 & 18 & 3 & 0 & 4 & 9 & 5 & 1 & 6 & . & 7 & . & 8 & . & 9 & . & 10 & 1 & 11 & 0\end{array}$ $\begin{array}{llllllllllllllllllllllllll}44 & 2 & 3 & 10 & 1 & 7 & 2 & 3 & 3 & 0 & 4 & 18 & 5 & 10 & 6 & 5 & 7 & 0 & 8 & 0 & 9 & 0 & 10 & 0 & 11 & .\end{array}$ $\begin{array}{llllllllllllllllllllllllll}45 & 2 & 3 & 15 & 1 & 0 & 2 & 0 & 3 & 0 & 4 & 4 & 5 & 0 & 6 & 0 & 7 & 0 & 8 & 3 & 9 & 1 & 10 & 0 & 11 & 1\end{array}$ $\begin{array}{llllllllllllllllllllllllll}46 & 2 & 3 & 17 & 1 & 3 & 2 & 12 & 3 & 1 & 4 & 6 & 5 & 4 & 6 & 1 & 7 & 1 & 8 & 2 & 9 & 1 & 10 & 0 & 11 & 0\end{array}$ $\begin{array}{llllllllllllllllllllllllll}47 & 2 & 3 & 21 & 1 & 3 & 2 & 15 & 3 & 1 & 4 & 3 & 5 & 1 & 6 & 1 & 7 & 0 & 8 & 1 & 9 & 0 & 10 & 0 & 11 & .\end{array}$ $\begin{array}{llllllllllllllllllllllllll}48 & 2 & 3 & 23 & 1 & 1 & 2 & 22 & 3 & 0 & 4 & 10 & 5 & 8 & 6 & 0 & 7 & 0 & 8 & 4 & 9 & 0 & 10 & 0 & 11 & 0\end{array}$ $\begin{array}{llllllllllllllllllllllllll}49 & 2 & 3 & 26 & 1 & 1 & 2 & 27 & 3 & 1 & 4 & 8 & 5 & 4 & 6 & 1 & 7 & 0 & 8 & 0 & 9 & 0 & 10 & 1 & 11 & 0\end{array}$ $\begin{array}{llllllllllllllllllllllllll}\mathbf{5 0} & 2 & \mathbf{3} & \mathbf{3 0} & \mathbf{1} & \mathbf{2} & \mathbf{2} & \mathbf{8} & \mathbf{3} & \mathbf{1} & \mathbf{4} & \mathbf{8} & \mathbf{5} & \mathbf{6} & \mathbf{6} & \mathbf{0} & \mathbf{7} & \mathbf{0} & \mathbf{8} & \mathbf{0} & \mathbf{9} & \mathbf{0} & \mathbf{1 0} & \mathbf{0} & \mathbf{1 1} & \mathbf{0}\end{array}$ $\begin{array}{lllllllllllllllllllllllllll}51 & 2 & 3 & 31 & 1 & 5 & 2 & 13 & 3 & 4 & 4 & 6 & 5 & 8 & 6 & 1 & 7 & 2 & 8 & 7 & 9 & 1 & 10 & 1 & 11 & 0\end{array}$ $\begin{array}{llllllllllllllllllllllllll}52 & 2 & 3 & 36 & 1 & 0 & 2 & 31 & 3 & 0 & 4 & 11 & 5 & 13 & 6 & 0 & 7 & 0 & 8 & 9 & 9 & 0 & 10 & 5 & 11 & 1\end{array}$ $\begin{array}{llllllllllllllllllllllllll}53 & 2 & 3 & 42 & 1 & 1 & 2 & 5 & 3 & 2 & 4 & 10 & 5 & 17 & 6 & 1 & 7 & 0 & 8 & 4 & 9 & 0 & 10 & 0 & 11 & 2\end{array}$ $\begin{array}{llllllllllllllllllllllllll}54 & 2 & 3 & 44 & 1 & 1 & 2 & 16 & 3 & 0 & 4 & 8 & 5 & 8 & 6 & 2 & 7 & 0 & 8 & 5 & 9 & 0 & 10 & 0 & 11 & 0\end{array}$ $\begin{array}{llllllllllllllllllllllllll}55 & 2 & 3 & 47 & 1 & 0 & 2 & 14 & 3 & 0 & 4 & 2 & 5 & 0 & 6 & 0 & 7 & 0 & 8 & 1 & 9 & 0 & 10 & 0 & 11 & 0\end{array}$ $\begin{array}{llllllllllllllllllllllllll}56 & 2 & 3 & 48 & 1 & 4 & 2 & 9 & 3 & 0 & 4 & 12 & 5 & 6 & 6 & 0 & 7 & 0 & 8 & 1 & 9 & 1 & 10 & 1 & 11 & 2\end{array}$ $\begin{array}{llllllllllllllllllllllllll}57 & 2 & 3 & 53 & 1 & 2 & 2 & 18 & 3 & 3 & 4 & 2 & 5 & 1 & 6 & 0 & 7 & 0 & 8 & 2 & 9 & 0 & 10 & 0 & 11 & 0\end{array}$ $\begin{array}{lllllllllllllllllllllllllll}58 & 2 & 3 & 56 & 1 & 0 & 2 & 14 & 3 & 0 & 4 & 5 & 5 & 4 & 6 & 0 & 7 & 0 & 8 & 0 & 9 & 0 & 10 & 3 & 11 & 0\end{array}$ $\begin{array}{llllllllllllllllllllllllllll}59 & 2 & 3 & 57 & 1 & 1 & 2 & 9 & 3 & 0 & 4 & 2 & 5 & 5 & 6 & 0 & 7 & 0 & 8 & 0 & 9 & 0 & 10 & 6 & 11 & 17\end{array}$ $\begin{array}{llllllllllllllllllllllllll}60 & 2 & 3 & 60 & 1 & 0 & 2 & 32 & 3 & 0 & 4 & 1 & 5 & 1 & 6 & 3 & 7 & 0 & 8 & 2 & 9 & 0 & 10 & 3 & 11 & 1\end{array}$ $\begin{array}{llllllllllllllllllllllllll}61 & 2 & 4 & 3 & 1 & 0 & 2 & 26 & 3 & 0 & 4 & 8 & 5 & 11 & 6 & 3 & 7 & 0 & 8 & 5 & 9 & 0 & 10 & 0 & 11 & 0\end{array}$ $\begin{array}{llllllllllllllllllllllllll}62 & 2 & 4 & 4 & 1 & 3 & 2 & 18 & 3 & 3 & 4 & 4 & 5 & 9 & 6 & 0 & 7 & 1 & 8 & 6 & 9 & 0 & 10 & 8 & 11 & 2\end{array}$ $\begin{array}{llllllllllllllllllllllllll}63 & 2 & 4 & 6 & 1 & 1 & 2 & 19 & 3 & 0 & 4 & 8 & 5 & 11 & 6 & 3 & 7 & 0 & 8 & 2 & 9 & 0 & 10 & 2 & 11 & 7\end{array}$ $\begin{array}{llllllllllllllllllllllllllll}64 & 2 & 4 & 12 & 1 & 1 & 2 & 13 & 3 & 0 & 4 & 7 & 5 & 3 & 6 & 0 & 7 & 1 & 8 & 4 & 9 & 0 & 10 & 2 & 11 & 0\end{array}$ 


\begin{tabular}{|c|c|c|c|c|c|c|c|c|c|c|c|c|c|c|c|c|c|c|c|c|c|c|c|c|c|}
\hline 65 & 2 & 4 & 13 & 1 & 0 & 2 & 5 & 3 & 0 & 4 & 2 & 5 & 4 & 6 & 0 & 7 & 0 & 8 & 1 & 9 & 0 & 10 & 4 & 11 & 11 \\
\hline 66 & 2 & 4 & 15 & 1 & 1 & 2 & 5 & 3 & 0 & 4 & 1 & 5 & 5 & 6 & 0 & 7 & 0 & 8 & 0 & 9 & 1 & 10 & 0 & 11 & $\mathbf{0}$ \\
\hline 67 & 2 & 4 & 19 & 1 & 0 & 2 & 5 & 3 & 0 & 4 & 9 & 5 & 7 & 6 & $\mathbf{0}$ & 7 & 0 & 8 & 2 & 9 & $\boldsymbol{1}$ & 10 & 10 & 11 & 4 \\
\hline 68 & 2 & 4 & 21 & 1 & 4 & 2 & 2 & 3 & 0 & 4 & 10 & 5 & 8 & 6 & 2 & 7 & 0 & 8 & 0 & 9 & $\mathbf{I}$ & 10 & 10 & 11 & 9 \\
\hline 69 & 2 & 4 & 26 & 1 & 2 & 2 & 0 & 3 & 2 & 4 & 3 & 5 & 2 & 6 & 0 & 7 & 2 & 8 & 0 & 9 & (0) & 10 & 3 & 11 & 3 \\
\hline 70 & 2 & 4 & 27 & 1 & 1 & 2 & 3 & 3 & 1 & 4 & 6 & 5 & 11 & 6 & 1 & 7 & 0 & 8 & 0 & 9 & 0 & 10 & 2 & 11 & 0 \\
\hline 71 & 2 & 4 & 31 & 1 & 2 & 2 & 29 & 3 & 0 & 4 & 5 & 5 & $\mathbf{0}$ & 6 & $\mathbf{0}$ & 7 & 0 & 8 & $\mathbf{0}$ & 9 & 2 & 10 & 1 & 11 & 2 \\
\hline 72 & 2 & 4 & 32 & 1 & 4 & 2 & 22 & 3 & 1 & 4 & 0 & 5 & 8 & 6 & 0 & 7 & 0 & 8 & 0 & 9 & 0 & 10 & 0 & 11 & 11 \\
\hline 73 & 2 & 4 & 37 & 1 & 5 & 2 & 1 & 3 & 0 & 4 & 2 & 5 & 5 & 6 & 1 & 7 & 0 & 8 & 1 & 9 & 0 & 10 & 12 & 11 & 1 \\
\hline 74 & 2 & 4 & 43 & 1 & 6 & 2 & 7 & 3 & 2 & 4 & 1 & 5 & 2 & 6 & 2 & 7 & 0 & 8 & 0 & 9 & 0 & 10 & 4 & 11 & 2 \\
\hline 75 & 2 & 4 & 45 & 1 & 2 & 2 & 2 & 3 & 0 & 4 & 3 & 5 & 2 & 6 & 1 & 7 & 2 & 8 & 3 & 9 & ( & 10 & 7 & 11 & 2 \\
\hline 76 & 2 & 4 & 48 & 1 & 0 & 2 & 21 & 3 & 1 & 4 & 4 & 5 & 3 & 6 & 1 & 7 & 2 & 8 & 0 & 9 & 0 & 10 & 0 & 11 & 1 \\
\hline 77 & 2 & 4 & 49 & 1 & 2 & 2 & 38 & 3 & 1 & 4 & 16 & 5 & 4 & 6 & 5 & 7 & 3 & 8 & 8 & 9 & 0 & 10 & 0 & 11 & 0 \\
\hline 78 & 2 & 4 & 54 & 1 & 0 & 2 & 18 & 3 & 0 & 4 & 1 & 5 & . & 6 & . & 7 & . & 8 & . & 9 & • & 10 & . & 11 & . \\
\hline 79 & 2 & 4 & 55 & 1 & 1 & 2 & 18 & 3 & 1 & 4 & 5 & 5 & 1 & 6 & $\mathbf{0}$ & 7 & 3 & 8 & 2 & 9 & 3 & 10 & 1 & 11 & 13 \\
\hline 80 & 2 & 4 & 51 & 1 & 0 & 2 & 7 & 3 & 3 & 4 & 2 & 5 & . & 6 & . & 7 & . & 8 & . & 9 & & 10 & • & 11 & \\
\hline
\end{tabular}

ods html close; /* close previous $* /$

ods html; /* open new */;

ods html;

ods graphics on ;

proc glimmix data $=$ rostrent;

class plotid block time;

model count $1=$ time $/$ dist $=$ poisson htype $=3$;

Random block $/$ subject $=$ plotid type $=\operatorname{ar}(1)$;

covtest glm / classical ; 
$*_{\text {output } \text { out }=\text { Residuals }}$

* pred $=$ Pred

* resraw = Resraw

* $\quad$ reschi $=$ Reschi

* resdev $=$ Resdev

* $\quad$ stdreschi $=$ Stdreschi

* $\quad$ stdresdev $=$ Stdresdev

* $\quad$ reslik = Reslik;

Lsmeans time/ilink pdiff adjust = tukey;

*proc sort;

*by time;

*proc means n mean std stderr min max;

*by time;

*var count;

run;

proc sort;

by time;

proc means $n$ mean std stderr min max;

by time;

var count;

run; 
/*Data are mature plants, plotid, counts, quadrats. There are two woods each with two blocks. Quadno are quadrat numbers, numbers 1-11 indicate sample years from 2004-

2014.

Codes are SAS for PROC GLIMMIX.*/

Data maturplt;

Input plotid woods block quadno @;

do $\mathrm{i}=1$ to 11 ;

input time count @@;

count $1=($ count +1$)$;

output;

end;

datalines;

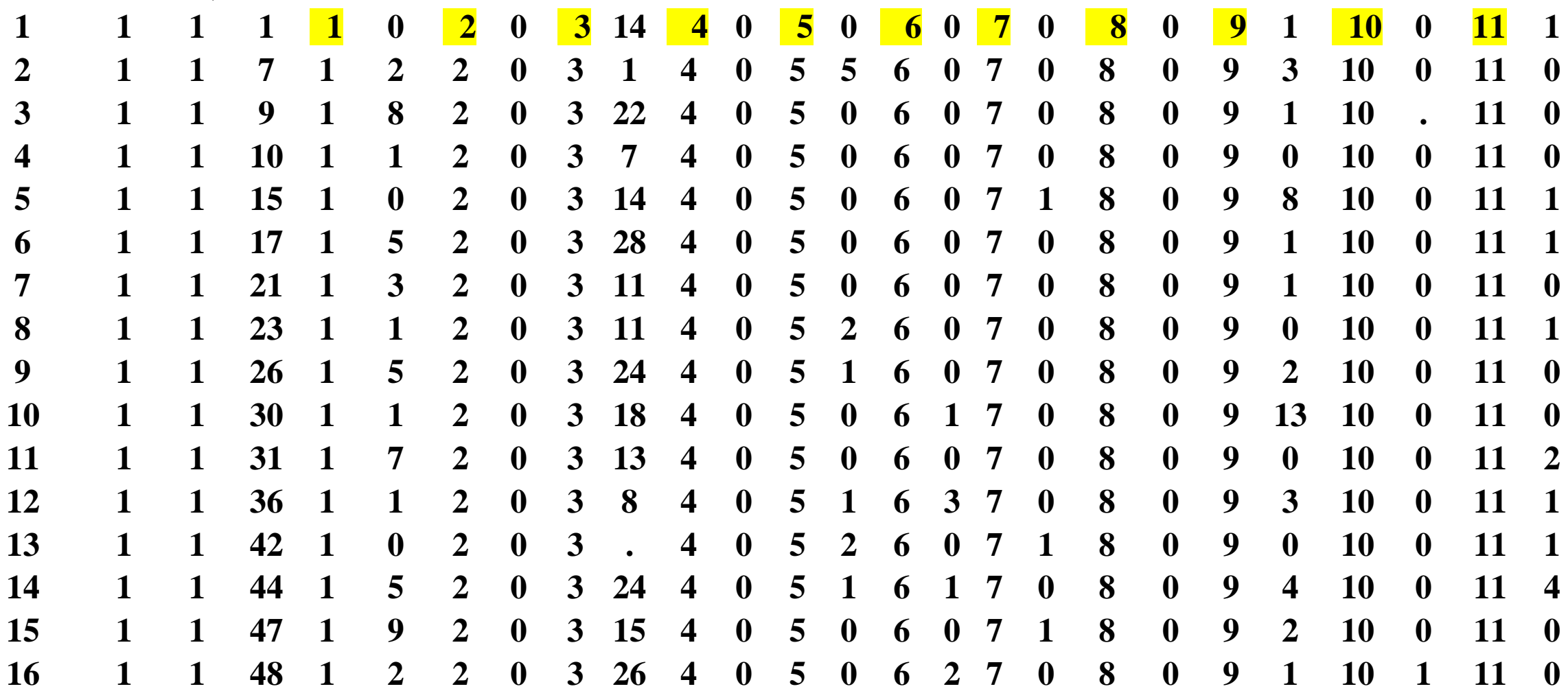


$\begin{array}{llllllllllllllllllllllllll}17 & 1 & 1 & 53 & 1 & 1 & 2 & 0 & 3 & 22 & 4 & 0 & 5 & 0 & 6 & 0 & 7 & 1 & 8 & 0 & 9 & 1 & 10 & 1 & 11 & 0\end{array}$ $\begin{array}{llllllllllllllllllllllllll}18 & 1 & 1 & 56 & 1 & 9 & 2 & 0 & 3 & 15 & 4 & 0 & 5 & 2 & 6 & 0 & 7 & 1 & 8 & 0 & 9 & 0 & 10 & 0 & 11 & 0\end{array}$ $\begin{array}{lllllllllllllllllllllllllll}19 & 1 & 1 & 57 & 1 & 6 & 2 & 0 & 3 & 20 & 4 & 0 & 5 & 0 & 6 & 0 & 7 & 0 & 8 & 0 & 9 & 1 & 10 & 0 & 11 & 0\end{array}$ $\begin{array}{llllllllllllllllllllllllll}20 & 1 & 1 & 60 & 1 & 3 & 2 & 0 & 3 & 8 & 4 & 0 & 5 & 0 & 6 & 0 & 7 & 0 & 8 & 0 & 9 & 0 & 10 & 0 & 11 & 0\end{array}$ $\begin{array}{llllllllllllllllllllllllll}21 & 1 & 2 & 1 & 1 & 0 & 2 & 3 & 3 & 5 & 4 & 0 & 5 & 1 & 6 & 0 & 7 & 1 & 8 & 0 & 9 & 3 & 10 & 0 & 11 & 0\end{array}$ $\begin{array}{llllllllllllllllllllllllll}22 & 1 & 2 & 7 & 1 & 3 & 2 & 0 & 3 & 9 & 4 & 0 & 5 & 0 & 6 & 0 & 7 & 0 & 8 & 0 & 9 & 1 & 10 & 0 & 11 & 0\end{array}$ $\begin{array}{llllllllllllllllllllllllll}23 & 1 & 2 & 9 & 1 & 0 & 2 & 0 & 3 & 12 & 4 & 0 & 5 & 0 & 6 & 1 & 7 & 0 & 8 & 0 & 9 & 1 & 10 & 1 & 11 & 0\end{array}$ $\begin{array}{llllllllllllllllllllllllll}24 & 1 & 2 & 10 & 1 & 0 & 2 & 1 & 3 & 15 & 4 & 0 & 5 & 0 & 6 & 0 & 7 & 0 & 8 & 0 & 9 & 0 & 10 & 0 & 11 & 0\end{array}$ $\begin{array}{llllllllllllllllllllllllll}25 & 1 & 2 & 15 & 1 & 3 & 2 & 0 & 3 & 18 & 4 & 0 & 5 & 0 & 6 & 0 & 7 & 3 & 8 & 1 & 9 & 4 & 10 & 1 & 11 & 0\end{array}$ $\begin{array}{llllllllllllllllllllllllll}26 & 1 & 2 & 17 & 1 & 4 & 2 & 0 & 3 & 15 & 4 & 0 & 5 & 0 & 6 & 0 & 7 & 1 & 8 & 0 & 9 & 1 & 10 & 0 & 11 & 0\end{array}$ $\begin{array}{llllllllllllllllllllllllll}27 & 1 & 2 & 21 & 1 & 0 & 2 & 0 & 3 & 0 & 4 & 1 & 5 & 2 & 6 & 1 & 7 & 0 & 8 & 0 & 9 & 2 & 10 & 0 & 11 & 0\end{array}$ $\begin{array}{llllllllllllllllllllllllll}28 & 1 & 2 & 23 & 1 & 0 & 2 & 1 & 3 & 24 & 4 & 0 & 5 & 0 & 6 & 0 & 7 & 0 & 8 & 0 & 9 & 3 & 10 & 0 & 11 & 0\end{array}$ $\begin{array}{llllllllllllllllllllllllll}29 & 1 & 2 & 26 & 1 & 3 & 2 & 0 & 3 & 33 & 4 & 0 & 5 & 0 & 6 & 0 & 7 & 0 & 8 & 0 & 9 & 1 & 10 & 0 & 11 & 0\end{array}$ $\begin{array}{llllllllllllllllllllllllll}30 & 1 & 2 & 30 & 1 & 0 & 2 & 0 & 3 & 4 & 4 & 0 & 5 & 2 & 6 & 0 & 7 & 0 & 8 & 0 & 9 & 8 & 10 & 0 & 11 & 0\end{array}$ $\begin{array}{llllllllllllllllllllllllll}31 & 1 & 2 & 31 & 1 & 0 & 2 & 0 & 3 & 3 & 4 & 0 & 5 & 0 & 6 & 0 & 7 & 2 & 8 & 0 & 9 & 0 & 10 & 0 & 11 & 0\end{array}$ $\begin{array}{llllllllllllllllllllllllll}32 & 1 & 2 & 36 & 1 & 0 & 2 & 0 & 3 & 10 & 4 & 0 & 5 & 0 & 6 & 1 & 7 & 0 & 8 & 1 & 9 & 4 & 10 & 1 & 11 & 0\end{array}$ $\begin{array}{llllllllllllllllllllllllll}33 & 1 & 2 & 42 & 1 & 1 & 2 & 2 & 3 & 27 & 4 & 0 & 5 & 2 & 6 & 0 & 7 & 0 & 8 & 0 & 9 & 0 & 10 & 0 & 11 & 0\end{array}$ $\begin{array}{llllllllllllllllllllllllll}34 & 1 & 2 & 44 & 1 & 1 & 2 & 0 & 3 & 9 & 4 & 0 & 5 & 4 & 6 & 0 & 7 & 0 & 8 & 0 & 9 & 1 & 10 & 0 & 11 & 0\end{array}$ $\begin{array}{lllllllllllllllllllllllllll}35 & 1 & 2 & 47 & 1 & 11 & 2 & 0 & 3 & 18 & 4 & 0 & 5 & 0 & 6 & 0 & 7 & 0 & 8 & 0 & 9 & 0 & 10 & 0 & 11 & 0\end{array}$ $\begin{array}{llllllllllllllllllllllllll}36 & 1 & 2 & 48 & 1 & 0 & 2 & 2 & 3 & 4 & 4 & 0 & 5 & 0 & 6 & 0 & 7 & 0 & 8 & 0 & 9 & 0 & 10 & 0 & 11 & 0\end{array}$ $\begin{array}{llllllllllllllllllllllllll}37 & 1 & 2 & 53 & 1 & 3 & 2 & 0 & 3 & 25 & 4 & 0 & 5 & 2 & 6 & 0 & 7 & 3 & 8 & 0 & 9 & 2 & 10 & 1 & 11 & 0\end{array}$ $\begin{array}{llllllllllllllllllllllllll}38 & 1 & 2 & 56 & 1 & 2 & 2 & 0 & 3 & 15 & 4 & 0 & 5 & 1 & 6 & 1 & 7 & 1 & 8 & 0 & 9 & 0 & 10 & 0 & 11 & 0\end{array}$ $\begin{array}{llllllllllllllllllllllllll}39 & 1 & 2 & 57 & 1 & 16 & 2 & 0 & 3 & 17 & 4 & 0 & 5 & 1 & 6 & 0 & 7 & 2 & 8 & 0 & 9 & 0 & 10 & 0 & 11 & 0\end{array}$ $\begin{array}{llllllllllllllllllllllllllll}40 & 1 & 2 & 60 & 1 & 0 & 2 & 0 & 3 & . & 4 & 0 & 5 & 0 & 6 & 0 & 7 & 0 & 8 & 0 & 9 & 1 & 10 & 0 & 11 & 0\end{array}$ $\begin{array}{llllllllllllllllllllllllll}41 & 2 & 3 & 1 & 1 & 0 & 2 & 0 & 3 & 3 & 4 & 0 & 5 & 3 & 6 & 0 & 7 & 0 & 8 & 0 & 9 & 0 & 10 & 1 & 11 & 0\end{array}$ $\begin{array}{llllllllllllllllllllllllll}42 & 2 & 3 & 7 & 1 & 7 & 2 & 0 & 3 & 18 & 4 & 0 & 5 & 0 & 6 & 0 & 7 & 1 & 8 & 0 & 9 & 0 & 10 & 0 & 11 & 0\end{array}$ $\begin{array}{lllllllllllllllllllllllllllll}43 & 2 & 3 & 9 & 1 & 1 & 2 & 0 & 3 & 9 & 4 & 0 & 5 & 0 & 6 & . & 7 & . & 8 & . & 9 & . & 10 & 0 & 11 & 0\end{array}$ $\begin{array}{llllllllllllllllllllllllll}44 & 2 & 3 & 10 & 1 & 1 & 2 & 0 & 3 & 4 & 4 & 0 & 5 & 0 & 6 & 0 & 7 & 0 & 8 & 0 & 9 & 0 & 10 & 0 & 11 & .\end{array}$ $\begin{array}{llllllllllllllllllllllllll}45 & 2 & 3 & 15 & 1 & 0 & 2 & 0 & 3 & 3 & 4 & 0 & 5 & 1 & 6 & 0 & 7 & 0 & 8 & 0 & 9 & 0 & 10 & 1 & 11 & 0\end{array}$ $\begin{array}{llllllllllllllllllllllllll}46 & 2 & 3 & 17 & 1 & 5 & 2 & 0 & 3 & 12 & 4 & 0 & 5 & 1 & 6 & 0 & 7 & 0 & 8 & 0 & 9 & 1 & 10 & 0 & 11 & 0\end{array}$ 
$\begin{array}{llllllllllllllllllllllllll}47 & 2 & 3 & 21 & 1 & 5 & 2 & 1 & 3 & 8 & 4 & 0 & 5 & 2 & 6 & 0 & 7 & 0 & 8 & 0 & 9 & 0 & 10 & 0 & 11 & .\end{array}$ $\begin{array}{llllllllllllllllllllllllll}48 & 2 & 3 & 23 & 1 & 7 & 2 & 0 & 3 & 23 & 4 & 0 & 5 & 0 & 6 & 1 & 7 & 0 & 8 & 0 & 9 & 0 & 10 & 0 & 11 & 0\end{array}$ $\begin{array}{llllllllllllllllllllllllll}49 & 2 & 3 & 26 & 1 & 4 & 2 & 0 & 3 & 7 & 4 & 0 & 5 & 1 & 6 & 0 & 7 & 0 & 8 & 0 & 9 & 0 & 10 & 0 & 11 & 0\end{array}$ $\begin{array}{llllllllllllllllllllllllll}\mathbf{5 0} & \mathbf{2} & \mathbf{3} & \mathbf{3 0} & \mathbf{1} & \mathbf{5} & \mathbf{2} & \mathbf{0} & \mathbf{3} & \mathbf{1} & \mathbf{4} & \mathbf{0} & \mathbf{5} & \mathbf{0} & \mathbf{6} & \mathbf{0} & \mathbf{7} & \mathbf{2} & \mathbf{8} & \mathbf{0} & \mathbf{9} & \mathbf{1} & \mathbf{1 0} & \mathbf{0} & \mathbf{1 1} & \mathbf{0}\end{array}$ $\begin{array}{lllllllllllllllllllllllllll}51 & 2 & 3 & 31 & 1 & 6 & 2 & 0 & 3 & 8 & 4 & 0 & 5 & 0 & 6 & 0 & 7 & 4 & 8 & 0 & 9 & 0 & 10 & 3 & 11 & 0\end{array}$ $\begin{array}{llllllllllllllllllllllllll}52 & 2 & 3 & 36 & 1 & 24 & 2 & 0 & 3 & 16 & 4 & 0 & 5 & 0 & 6 & 0 & 7 & 4 & 8 & 0 & 9 & 0 & 10 & 0 & 11 & 0\end{array}$ $\begin{array}{llllllllllllllllllllllllll}53 & 2 & 3 & 42 & 1 & 6 & 2 & 0 & 3 & 20 & 4 & 0 & 5 & 0 & 6 & 0 & 7 & 0 & 8 & 0 & 9 & 0 & 10 & 0 & 11 & 0\end{array}$ $\begin{array}{lllllllllllllllllllllllllll}54 & 2 & 3 & 44 & 1 & 9 & 2 & 0 & 3 & 18 & 4 & 0 & 5 & 1 & 6 & 0 & 7 & 2 & 8 & 0 & 9 & 0 & 10 & 0 & 11 & 0\end{array}$ $\begin{array}{llllllllllllllllllllllllll}55 & 2 & 3 & 47 & 1 & 4 & 2 & 0 & 3 & 12 & 4 & 0 & 5 & 1 & 6 & 0 & 7 & 0 & 8 & 0 & 9 & 0 & 10 & 1 & 11 & 0\end{array}$ $\begin{array}{llllllllllllllllllllllllll}56 & 2 & 3 & 48 & 1 & 17 & 2 & 0 & 3 & 12 & 4 & 0 & 5 & 0 & 6 & 0 & 7 & 3 & 8 & 0 & 9 & 0 & 10 & 1 & 11 & 0\end{array}$ $\begin{array}{llllllllllllllllllllllllll}57 & 2 & 3 & 53 & 1 & 9 & 2 & 0 & 3 & 2 & 4 & 0 & 5 & 1 & 6 & 0 & 7 & 0 & 8 & 0 & 9 & 0 & 10 & 0 & 11 & 0\end{array}$ $\begin{array}{llllllllllllllllllllllllll}58 & 2 & 3 & 56 & 1 & 0 & 2 & 0 & 3 & 18 & 4 & 0 & 5 & 0 & 6 & 0 & 7 & 0 & 8 & 0 & 9 & 0 & 10 & 0 & 11 & 0\end{array}$ $\begin{array}{llllllllllllllllllllllllll}59 & 2 & 3 & 57 & 1 & 4 & 2 & 0 & 3 & 10 & 4 & 0 & 5 & 0 & 6 & 1 & 7 & 0 & 8 & 0 & 9 & 0 & 10 & 1 & 11 & 0\end{array}$ $\begin{array}{llllllllllllllllllllllllll}60 & 2 & 3 & 60 & 1 & 11 & 2 & 0 & 3 & 10 & 4 & 0 & 5 & 2 & 6 & 0 & 7 & 0 & 8 & 0 & 9 & 2 & 10 & 4 & 11 & 0\end{array}$ $\begin{array}{llllllllllllllllllllllllll}61 & 2 & 4 & 3 & 1 & 9 & 2 & 0 & 3 & 13 & 4 & 0 & 5 & 0 & 6 & 2 & 7 & 1 & 8 & 0 & 9 & 0 & 10 & 0 & 11 & 0\end{array}$ $\begin{array}{llllllllllllllllllllllllll}62 & 2 & 4 & 4 & 1 & 6 & 2 & 0 & 3 & 14 & 4 & 0 & 5 & 0 & 6 & 1 & 7 & 0 & 8 & 1 & 9 & 1 & 10 & 0 & 11 & 0\end{array}$ $\begin{array}{llllllllllllllllllllllllll}63 & 2 & 4 & 6 & 1 & 5 & 2 & 0 & 3 & 7 & 4 & 0 & 5 & 0 & 6 & 0 & 7 & 0 & 8 & 0 & 9 & 1 & 10 & 1 & 11 & 1\end{array}$ $\begin{array}{llllllllllllllllllllllllll}64 & 2 & 4 & 12 & 1 & 6 & 2 & 0 & 3 & 13 & 4 & 0 & 5 & 0 & 6 & 1 & 7 & 1 & 8 & 0 & 9 & 0 & 10 & 0 & 11 & 0\end{array}$ $\begin{array}{llllllllllllllllllllllllll}65 & 2 & 4 & 13 & 1 & 3 & 2 & 0 & 3 & 14 & 4 & 0 & 5 & 0 & 6 & 0 & 7 & 0 & 8 & 0 & 9 & 1 & 10 & 1 & 11 & 1\end{array}$ $\begin{array}{lllllllllllllllllllllllllll}66 & 2 & 4 & 15 & 1 & 0 & 2 & 0 & 3 & 9 & 4 & 0 & 5 & 0 & 6 & 0 & 7 & 1 & 8 & 0 & 9 & 0 & 10 & 1 & 11 & 0\end{array}$ $\begin{array}{llllllllllllllllllllllllll}67 & 2 & 4 & 19 & 1 & 4 & 2 & 0 & 3 & 16 & 4 & 0 & 5 & 0 & 6 & 2 & 7 & 0 & 8 & 0 & 9 & 0 & 10 & 0 & 11 & 0\end{array}$ $\begin{array}{llllllllllllllllllllllllll}68 & 2 & 4 & 21 & 1 & 0 & 2 & 0 & 3 & 18 & 4 & 0 & 5 & 0 & 6 & 0 & 7 & 0 & 8 & 0 & 9 & 7 & 10 & 1 & 11 & 0\end{array}$ $\begin{array}{llllllllllllllllllllllllll}69 & 2 & 4 & 26 & 1 & 4 & 2 & 3 & 3 & 2 & 4 & 0 & 5 & 0 & 6 & 3 & 7 & 0 & 8 & 0 & 9 & 4 & 10 & 0 & 11 & 0\end{array}$ $\begin{array}{lllllllllllllllllllllllllll}\mathbf{7 0} & 2 & \mathbf{2} & \mathbf{2 7} & \mathbf{1} & \mathbf{3} & \mathbf{2} & \mathbf{0} & \mathbf{3} & \mathbf{6} & \mathbf{4} & \mathbf{1} & \mathbf{5} & \mathbf{3} & \mathbf{6} & \mathbf{0} & \mathbf{7} & \mathbf{0} & \mathbf{8} & \mathbf{0} & \mathbf{9} & \mathbf{2} & \mathbf{1 0} & \mathbf{0} & \mathbf{1 1} & \mathbf{2}\end{array}$ $\begin{array}{llllllllllllllllllllllllll}71 & 2 & 4 & 31 & 1 & 8 & 2 & 0 & 3 & 53 & 4 & 0 & 5 & 0 & 6 & 0 & 7 & 0 & 8 & 3 & 9 & 0 & 10 & 8 & 11 & 0\end{array}$ $\begin{array}{llllllllllllllllllllllllll}72 & 2 & 4 & 32 & 1 & 2 & 2 & 0 & 3 & 25 & 4 & 0 & 5 & 0 & 6 & 0 & 7 & 0 & 8 & 0 & 9 & 0 & 10 & 0 & 11 & 0\end{array}$ $\begin{array}{llllllllllllllllllllllllll}73 & 2 & 4 & 37 & 1 & 1 & 2 & 0 & 3 & 4 & 4 & 0 & 5 & 0 & 6 & 0 & 7 & 2 & 8 & 0 & 9 & 3 & 10 & 0 & 11 & 0\end{array}$ $\begin{array}{llllllllllllllllllllllllll}74 & 2 & 4 & 43 & 1 & 2 & 2 & 2 & 3 & 13 & 4 & 0 & 5 & 0 & 6 & 0 & 7 & 2 & 8 & 0 & 9 & 0 & 10 & 0 & 11 & 1\end{array}$ $\begin{array}{llllllllllllllllllllllllll}75 & 2 & 4 & 45 & 1 & 2 & 2 & 0 & 3 & 10 & 4 & 0 & 5 & 0 & 6 & 2 & 7 & 0 & 8 & 1 & 9 & 0 & 10 & 10 & 11 & 0\end{array}$ $\begin{array}{llllllllllllllllllllllllll}76 & 2 & 4 & 48 & 1 & 10 & 2 & 1 & 3 & 15 & 4 & 0 & 5 & 0 & 6 & 2 & 7 & 0 & 8 & 0 & 9 & 0 & 10 & 4 & 11 & 0\end{array}$ 


$\begin{array}{cccccccccccccccccccccccccc}\mathbf{7 7} & \mathbf{2} & \mathbf{4} & \mathbf{4 9} & \mathbf{1} & \mathbf{9} & \mathbf{2} & \mathbf{0} & \mathbf{3} & \mathbf{1 8} & \mathbf{4} & \mathbf{0} & \mathbf{5} & \mathbf{3} & \mathbf{6} & \mathbf{0} & \mathbf{7} & \mathbf{0} & \mathbf{8} & \mathbf{0} & \mathbf{9} & \mathbf{1} & \mathbf{1 0} & \mathbf{0} & \mathbf{1 1} & \mathbf{0} \\ \mathbf{7 8} & \mathbf{2} & \mathbf{4} & \mathbf{5 4} & \mathbf{1} & \mathbf{1 3} & \mathbf{2} & \mathbf{0} & \mathbf{3} & \mathbf{4} & \mathbf{4} & \mathbf{0} & \mathbf{5} & . & \mathbf{6} & . & \mathbf{7} & . & \mathbf{8} & . & \mathbf{9} & . & \mathbf{1 0} & . & \mathbf{1 1} & . \\ \mathbf{7 9} & \mathbf{2} & \mathbf{4} & \mathbf{5 5} & \mathbf{1} & \mathbf{1 4} & \mathbf{2} & \mathbf{0} & \mathbf{3} & \mathbf{2} & \mathbf{4} & \mathbf{0} & \mathbf{5} & \mathbf{1} & \mathbf{6} & \mathbf{0} & \mathbf{7} & \mathbf{0} & \mathbf{8} & \mathbf{1} & \mathbf{9} & \mathbf{0} & \mathbf{1 0} & \mathbf{5} & \mathbf{1 1} & \mathbf{0} \\ \mathbf{8 0} & \mathbf{2} & \mathbf{4} & \mathbf{5 1} & \mathbf{1} & \mathbf{4} & \mathbf{2} & \mathbf{0} & \mathbf{3} & \mathbf{4} & \mathbf{4} & \mathbf{0} & \mathbf{5} & . & \mathbf{6} & . & \mathbf{7} & . & \mathbf{8} & . & \mathbf{9} & . & \mathbf{1 0} & . & \mathbf{1 1} & .\end{array}$

;

ods html close; /* close previous */

ods html; /* open new */;

ods html;

ods graphics on;

proc glimmix data $=$ maturplt ;

class plotid block time;

model count $1=$ time $/$ dist $=$ poisson htype $=3$;

Random block $/$ subject $=$ plotid type $=\operatorname{ar}(1)$;

covtest glm / classical ;

*output out = Residuals

$*$ pred $=$ Pred

$*$ resraw $=$ Resraw

* $\quad$ reschi $=$ Reschi

* resdev $=$ Resdev

* $\quad$ stdreschi $=$ Stdreschi

* $\quad$ stdresdev $=$ Stdresdev

* $\quad$ reslik = Reslik;

Lsmeans time/ilink pdiff adjust = tukey;

*proc sort;

*by time; 
*proc means $\mathrm{n}$ mean std stderr min max;

*by time;

*var count;

run;

proc sort;

by time;

proc means $n$ mean std stderr min max;

by time;

var count;

run; 


\section{Fig. S1}

Counts of 1st-yr rosettes and 2nd-yr mature flowering plants in control plots 2004-2014.

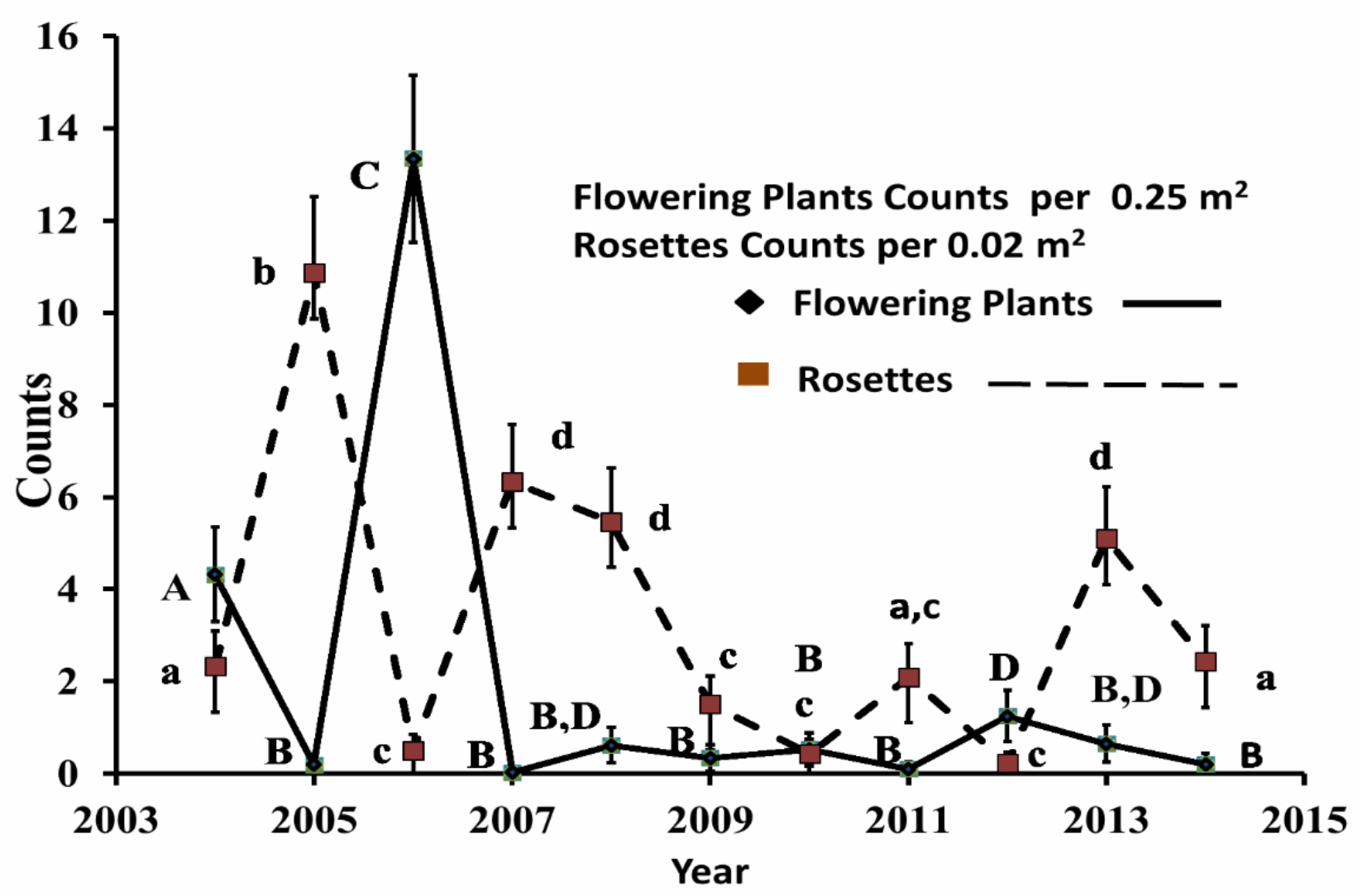

